\title{
Tratamiento de espasmos masivos con ACTH sintético
}

\author{
Isabel López S. ${ }^{* 1}$; Mónica Troncoso Sch. ${ }^{2}$; \\ Ledia Troncoso A. ${ }^{2}$; Fernando Novoa $S .^{2}$
}

\section{Treatment of infantile spasms with synthetic ACTH}

\begin{abstract}
Ten infants with infantile spasms (IS) and hypsarrhytmic EEG, received $0.5 \mathrm{mg}$ i.m. of synthetic ACTH, three tımes a week for 2 weeks. Six of them showed complete remission of seizures and dramatical EEG changes, and 3 cases resulted in a partial response. Spasms were controlled during the first week in $5 / 6$ cases where treatment was successful. A significant relationship was observed between treatment precocity and favourable outcome (Fisher $\mathrm{p}<0.02$ ). Synthetic ACTH treatment did not produce severe side effects in any patient. During follow up ( $\bar{X}$ $6.2 \mathrm{~m}$ ) relapse occurred in one case, with 5 patients maintaining the initial positive response and a normal or mildely retarded psychomotor development. The other five patients show severe psychomotor retardation and intractable spasms and/or other seizures. Future research should focuse on the efficacy of other treatment schedules. equili. brating success and side effects.

(Key words: Infantile spasms, treatment, ACTH.)
\end{abstract}

Los espasmos masivos (EM) son una forma severa de epilepsia del lactante, refractaria al tratamiento con los anticonvulsivantes habituales. Hace 30 affos se descubrió la eficacia de la corticotropina (ACTH) para controlar las crisis y mejorar el trazado electroencefalográfico ${ }^{3}$. Desde entonces se han empleado diferentes formas de tratamiento con ACTH y esteroides orales, obteniéndose una amplia variedad de resu]tados. Aunque en la actualidad el fármaco mas ampliamente aceptado es el ACTH natural gel, ACTH de origen animal de efecto prolongado, existe confusión en cuanto a dosis, esquema y duración de tratamiento ${ }^{2,3,4}$. Son factores claves en esta falta de consenso, una escasez de estudios prospectivos bien controlados y la diversidad de criterios empleados para evaluar la respuesta a tratamiento ${ }^{5}$.

Estudios bien controlados demuestran que al administrar ACTH gel por periodos de un mes, se logra control completo de las crisis en $70 \%$ de los casos ${ }^{6}$. Este planteamiento tiene

1. Unidad de Neuropsicología, Instituto de Nutrición y Tecnología de los Aljmentos, Universidad de Chile.

2. Servicio de Neuropsiquiatría Infantil, Hospital San Borja-Arriarán.

* Presentado por la primera autora como Trabajo de Ingreso a la Sociedad de Psiquiatría y Neurolog ía de la Infancin y Adolescencia en octubre de 1990. especial interés si se considera los serios efectos laterales que pueden presentarse, especialmente en curas prolongadas ${ }^{4,7}$, y apoya el uso de esquemas breves.

En nuestro medio no se dispone de ACTH natural gel, y los pacientes de EM son tratados con diferentes fármacos y esquemas, entre ellos betametasona y ACTH sintético de depósitos. Existen pocos antecedentes sobre el uso del ACTH sintético en EM. La mayoría de los autores lo desaconsejan porque produciría más efectos indeseables que el ACTH natural ${ }^{7,9-11}$. Sin embargo, en todos los trabajos aludidos se mencionan esquemas de administración diaria de 1 a $3 \mathrm{mg}$-dosis, sin considerar que los efectós laterales de ACTH son dosis dependien. tes y que los análogos sintéticos tienen un efecto más jntenso y prolongado que el gel de ACTH, utilizando como crjterio de apreciación la concentración de cortisol plasmático ${ }^{7,12}$.

A continuación presentamos los resultados de un estudio prospectivo cuyo propósito fue evaluar un esquema de tratamiento con ACTH sintético.

\section{Pacientes y Método}

Los criterios de selección de los pacjentes fueron los siguientes: evidencia clínica de crisis de EM; patrón electroencefalográfico de hipsartitmia, hipsarritmia mo- 
dificada o patrones ictales de hipsarritmia, según criterios de Gibbs ${ }^{13}$ y Hrachovy ${ }^{14}$ (figura 1); no haber recibido tratamiento previo con ACTH.

Ingresaron al estudio 10 pacientes, 8 varones y 2 niñas, referidos al Servicio de Neuropsiquiatría Infantil del Hospitai San Borja-Arriarán (ex Hospital Paula Jaraquemada), entre julio 1989 y junio 1990. La edad al ingreso estaba entre 5 y 17 meses, 6 eran menores de 1 afio ( $\bar{X}: 10,4$; d.e. : 4,5 meses). Los EM aparecieron entre los 2 y 14 meses de edad, siendo 9 casos menores de 1 año ( $\overline{\mathrm{X}}: 6,5$; d.e.: 3,5 meses). Ocho pacientes recibieron tratamiento previo, 5 con fenobarbital, 5 con ácido valproico, uno con nitrazepan y 4 con betametasona.

Todos los pacientes fueron estudiados según protocolo de pesquisa etiológica que se comunica en otro artículo de este volumen, catalogándose ocho de ellos como sintomáticos, con un factor etiológico identificado en siete casos, y dos como criptogenéticos (tabla 1 ). Se condicionó el comienzo del tratamiento con ACTH a la ausencia de signos elínicos de infección, controles basales de presión arterial, radiografía de tórax, hemograma, sedimento urinario y electroencefalograma (EEG). Adicionalmente se retiraron paulatinamente otros medicamentos anticonvulsivantes, a excepción de ácido valproico.

Todos los pacientes recibieron un esquema de tratamiento consistente en ACTH sintético de depósito (análogo sintétioo de ACTH que corresponde a la sécuencia aminoacídica 1.24 de la molécula original de ACTH de 39 aminoácidos, secuencia que posee la actividad biológica de la molécula. Esta sustancia se pre. senta en una suspensión con hidróxido de zinc, lo que determina su libexación lenta y efecto prolongado) ${ }^{15}$, en dosis de $0,5 \mathrm{mg}$ i.m., tres veces por semana, correspondientes a 0,046 a $0,063 \mathrm{mg} \times \mathrm{kg} \times$ dosis, durante dos semanas. Durante este período se controló peso y presión arterial y se efectuó un EEG finalizando la primera $y$ segunda semanas, todos informados por uno de los autores (L.T.) (tabla 1).

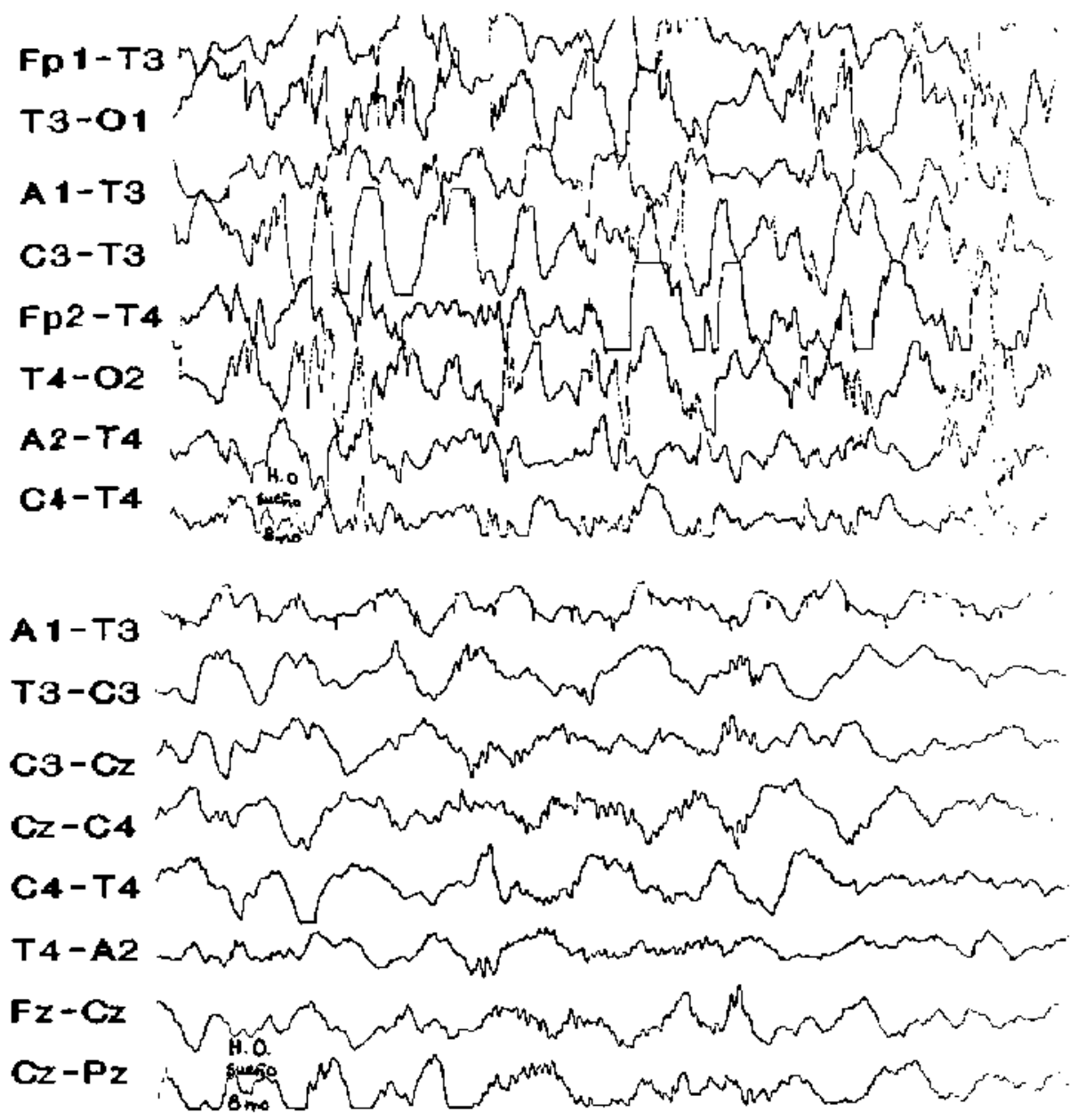

Figura 1: Trazado EEG durante el sueño, a) pre y b) postratamiento con ACTH sintético, observándose desaparición del patrón hipsarr ítmico (caso 4). 
Tabla 1

Diagnóstico, oportunidad y respuesta a tratamiento con ACTH sintético en 10 pacientes con espasmos masivos

\begin{tabular}{|c|c|c|}
\hline Factor etiológico & Lapso & Respuesta a \\
\hline $\begin{aligned} & \text { 1. } \text { Cristogenético } \\
& \text { 2. } \text { Genopatía } \\
& \text { 3. } \text { Enf. orina olor a jarabe de } \\
& \text { 4. } \text { arce variante } \\
& \text { Prematurez, hemorragia } \\
& \text { 5. } \text { intraventricular } \\
& \text { 6. } \text { Esclerosisa tuberosa } \\
& \text { 7. } \text { Criptogenético } \\
& \text { 8. } \text { Hiperlactatemia congénita } \\
& \text { 9. } \text { Sepsis neonatal, mictocefalia } \\
& \text { 10. } \text { Hemorragia intraparenquimatosa }\end{aligned}$ & $\begin{array}{r}2 \mathrm{~m} \\
12 \mathrm{~m} \\
1 \mathrm{~m} \\
1 \mathrm{~m} \\
3 \mathrm{~m} \\
1 \mathrm{~m} \\
5 \mathrm{~m} \\
1 \mathrm{~m} \\
14 \mathrm{~m} \\
2 \mathrm{sem}\end{array}$ & $\begin{array}{l}\text { Completa } \\
\text { Parcial } \\
\text { Completa } \\
\text { Completa } \\
\text { Parcial } \\
\text { Completa } \\
\text { No responde } \\
\text { Completa } \\
\text { Parcial } \\
\text { Completa }\end{array}$ \\
\hline
\end{tabular}

Se consideró tespuesta completa, el cese de los espasmos masjoos oon desaparición del patrón alterado en EEG (fígura 1) y como respuesta parcial a una teducción significatiya de las crisis con cambios electroencefalográficos menos llamativos.

Los resultados se analizaron estadísticamente utilizando ka prueba de probabilidades exactas de Fisher ${ }^{16}$.

\section{Resultados}

En la tabla 1 se resumen algunas características de los pacientes y su respuesta al tratamiento. Seis niños presentaron respuesta completa, tres parcial y en uno el trastorno no se modifi. co. Entre los que respondieron completamente, cínco lo hicieron durante la primera semana de tratamiento y uno durante la segunda semana. La djsminucjón, y en especial la desaparición de crisis, se asoció a cambios conductuales significativos expresados en mejor contacto con el medio y recuperación de habilidades en todas las areas de desarrollo. Cinco de los ocho pacientes sintomáticos y uno de los dos criptogenéticos respondieron en forma completa (N.S.). Se observó una tendencia que no alcanzó significacfón a una mejor respuesta en el grupo de niños cuyos EM comenzaron a los 7 meses o despues que en los menores de esa edad, cono asimismo en los que tenían mejor desarrollo inicial versus aquellos con retardo previo. Cuando el tiempo mediado entre el comienzo de EM y terapia con $\mathrm{ACTH}$ fue menor de un mes, todos los pacientes respondieron completamente; en cambio cuando la demora fue mayor, en sólo uno de cínco cesaron $(p<0,02)$ (tabla 2$)$.

Entre los efectos laterales de tratamiento se detectó ultrasonográficamente discreta dilatación ventricular en cuatro casos, que se jevertió en un plazo de 2 a 3 meses. En cinco pacientes se observó irritabilidad, manifestada por llanto frecuente y poca adaptación a rutinas, que cedió una vez finalizada la cura. No hubo casos de hipertensión arterial $o$ infecciones.

Estos ninos han sido seguidos por 6,2 meses en promedio con un margen de uno a doce meses, que en ocho de ellos es de cinco meses o más. Durante este tiempo en uno de los seis nifios con respuesta completa el paciente portador de hiperlactatemia congénita, quien tiene un perfil evolutivo de deterioro, reaparecieron las crisis. Los cinco casos restantes han sido seguidos entre 1-11 meses, sin presentar nuevamente espasmos u otras crisis, con desarrollo normal o retardo leve en cuatro casos. Todos reciben ácido valproico como tratamiento de mantención.

En tres casos con respuesta parcial, ésta se mantuvo por uno a tres meses pero posteriormente volvieron a reactivarse los espasmos. Todos estos pacientes tienen retardo severo del desarrollo. Por último, el paciente que no respondió ha sido también refractario a otros múltiples anticonvulsivantes y a dieta con triglicéridos de cadena mediana. 
Tabla 2

Respuesta a tratanuiento con ACTH sintético, según algunos factores relevantes

\begin{tabular}{|c|c|c|c|}
\hline & Completa & Parcial & s/Respuesta \\
\hline \multicolumn{4}{|l|}{ ETIOLOGIA } \\
\hline Criptogenético & $1 / 2$ & $0 / 2$ & $1 / 2$ \\
\hline Sintomático & $5 / 8$ & $\begin{array}{l}3 / 8 \\
\text { (N.S.) }\end{array}$ & - \\
\hline \multicolumn{4}{|l|}{ EDAD DE INICIO } \\
\hline$<7$ meses & $2 / 5$ & $3 / 5$ & - \\
\hline$>6$ meses & $4 / 5$ & $0 / 5$ & $1 / 5$ \\
\hline \multicolumn{4}{|c|}{ NIVEL DE DESARROLLO PREVIO } \\
\hline $\begin{array}{l}\text { Retraso seveto } \\
\text { Retraso moderado leve }\end{array}$ & $2 / 5$ & $3 / 5$ & - \\
\hline o normal & $4 / 5$ & $\begin{array}{l}0 / 5 \\
\text { (N.S.) }\end{array}$ & $1 / 5$ \\
\hline \multicolumn{4}{|c|}{ OPORTUNIDAD DE TRATAMIENTO } \\
\hline$<1$ mes & $5 / 5$ & - & - \\
\hline$>1$ mes & $1 / 5$ & $\begin{array}{c}3 / 5 \\
\text { (Fisher } \mathrm{p}<0,02 \text { ) }\end{array}$ & $1 / 5$ \\
\hline TOTAL & $6 / 10$ & $3 / 10$ & $1 / 10$ \\
\hline
\end{tabular}

\section{Comentario}

En este estudjo se definió como éxito de tratamiento al cese completo de los espasmos masi. vos con desaparición del patrón electroencefalográfico de hipsarritmia o formas modificadas de ella. Esta decisión se basó en la infommación obtenida en trabajos con monitoreo poligráfico y de video en que se demuestra que la respuesta a terapia hormonal, tanto al ACTH como esteroides orales, es del tipo "todo o nada"6, i", siendo este el criterio prevalente en la actualidad $^{4,5}$. Seis de diez de nuestros pacientes presentaron respuesta completa a tratamiento, man. teniéndose cinco de ellos libres de crisis hasta el momento ( $\overline{\mathrm{X}}: 6,4$ meses). Por el contrario, cuando se obtuvo una respuesta parcial, los pacientes volvieron a la situación inicial en dos meses. Nuestros resultados apoyan el criterio de respuesta "todo o nada", desaconsejando la definición de respuestas graduales.

El porcentaje de éxito de tratamiento alcanzado es semejante al de otras experiencias con administración de ACTH natural, sintético o esteroides orales en modalidades diversas ${ }^{2-5}$, si bien algo menor que en otros estudios en que se ha utilizado ACTH sintético en dosis y por tiempo significativamente mayores que en nuestro esquema $\mathrm{a}^{10, \mathrm{t}}$.

Todos los pacientes de este estudio recibieron la misma dosis de ACTH sintético, lo que produjo ligeras variaciones con respecto a la dosis por peso, sin que se observara, dentro de este rango, un efecto dosis respuesta. Algunos autores señalan que la probabilidad de éxito aumenta significativamente al administrar dosis elevadas, del order de $150 \mathrm{u} \times \mathrm{m}^{2} \mathrm{x}$ día $\left(3,75 \mathrm{mg} \cdot \mathrm{m}^{2}\right.$. día de ACTH sintético), pues producirían aumento sostenido del cortisol sérico ${ }^{12,18}$; sin embargo, otros estudios no han mostrado diferencias consistentes en cortisol sérico con dosis superiores a $20 \mathrm{u}$-día de ACTH, logrando buen éxito en $75 \%$ de los casos ${ }^{6,17,19}$. A diferencia de otras experiencias con ACTH sintético, el tratamiento se aplicó en días alternos, en consideración a las características especificas de este fámaco, lo que disminuye la probabilidad de efectos laterales y reduce el número de inyecciones intramusculares, obteniéndose resultados comparables a otras series. Es necesario, tanto en el aspecto de dosis unjtarias como el esquema de administración, generar experiencia en relación a la intensidad y duración del efecto de ACTH sintético en lactantes, a la vez que in- 
cluir dosis y esquemas como variables independientes en futuros diseños.

Nuestro tratamiento es uno de los más breves de los comunicados y en su disefio se consideró la información referente a momento de respues. ta $\mathrm{y}$ a los riesgos de tratamientos prolongados. Es conocido que las curas esteroidales prolongadas por meses implican un alto riesgo de severos efectos no deseados ${ }^{?, 10}$. Existe, en cambio, consenso en torno a curas más breves, fundamentados en que la respuesta completa se obtiene en la mayoría de los pacientes en las dos primeras semanas ${ }^{6,17}$. Algunos autores insisten en efectuar un retiro gradual hasta completar un periodo total de tres meses de tratamiento ${ }^{17}$. Otros, en cambio, han observado que cuando la respuesta es completa ésta se mantiene aun suspendiendo la cura, como ocurrió en la mayoría de nuestros casos ${ }^{6}$. Es probable que el estudio de un mayor número de pacientes y su seguimiento a largo plazo entregue evidencias mas sólidas en este sentido.

Las recaidas observadas no difieren de otras series que las describen en proporción de $30 \%$, la mayor parte en los dos a tres primeros meses después del tratamiento. Se estima que entre 74 y $100 \%$ de estos casos tesponden a una nueva cura con $\mathrm{ACTH}^{2}, 6,17,20$.

Diversos factores se han asociado a mayor probabilidad de éxito de tratamiento, entre ellos la etiología criptogenética, comienzo de los espasmos entre tres meses $y$ un año de edad, indemnidad neurológica inicial y precocidad del tratamiento esteroidal ${ }^{2-5,17,20}$. En nuestros pacientes no observamos diferencjas entre los grupos criptogenético y sintomátjco y sólo una tendencia a mejor respuesta en los casos de inicio más tardio o mejor nivel de desarrollo psicomotor inicial, lo que podria en parte atribuirse al pequeño tamaño de la muestra. En cambio, el tratamiento precoz fue significativamente más exitoso que el tardio. Esto podría deberse a mayor daño neurológico en los pacientes en que la enfermedad ha durado más tiempo, incidiendo en mayor refractariedad a tratamiento. Estos resultados apoyan la necesidad de emplear el ACTH precozmente.

Algunos estudjos han sefialado una incidencia relativamente alta de hipertensión arterial y dilatación del sistema ventricular como efectos no deseados de $\mathbf{A C T H}^{6,21}$. Otros han informado de $37 \%$ de efectos graves, entre ellos infecciones severas, hipertensión arterial, osteoporosis, altera- ciones hidroelectrolíticas y un $4 \%$ de mortalidad?. La gravedad $y$ frecuencia de ellos sería función de la dosis y duración del tratamiento, asi como del uso de ACTH sintético, en razón de una alteración más intensa y permanente del eje hipotálamo-hipófisis; sin embargo, nosotros no observamos hipertensión arterial o infecciones, aunque hubo un efecto de dilatación ventricular que fue reversible y efectos conductuales menores. Estos resultados sugieren que la administración de ACTH sintético en dosis de $0,05 \mathrm{mg} \cdot \mathbf{k g} \cdot$ dosis en días alternos y por períodos breves produce efectos laterales discretos, comparables a lo descrito en buenas series con ACTH natural ${ }^{5,6}$.

En síntesis, el diagnóstico clínico y electroencefalográfico de espasmos masivos en un lactante debe acompañarse de una decisión de tratamiento esteroidal en el corto plazo. El uso de ACTH sintético, disponjble en nuestro paîs, en una cura breve y en dias alternos, es efectivo en controlar completamente las crisis en aproximadamente la mitad de los casos. Este esquema, compañado de un estrecho control clínico, produce escasos efectos laterales en los niños. El éxito de tratamiento se acom. paña de un cambio conductual significativo y en algunos casos de recuperación de habilidades $y$ destrezas psicomotoras. Esto hace obligatorio, a nuestro juicio, intentar la cura esterojdal en todo paciente con EM.

Futuros estudios deberán aportar mayor información en torno a dosis mínima efectiva, esquema y duración de tratamiento, así como el impacto que éste tiene sobre el desarrollo neurológico y psicológico posterior de estos pacientes.

\section{Resumen}

Diez lactantes portadores de espasmos masivos (EM) e hipsarritmia recibieron un esquema de tratamiento con ACTH sintético 0,05 mg · $\mathrm{kg} \cdot \mathrm{do}$. sis 3 veces por semana por 2 semanas. En seis casos se obtuvo remisión completa de las crisis y cambios dramáticos en el electroencefalograma, en tres casos hubo una respuesta parcial. En cinco casos la respuesta completa ocurrió durante la primera semana de tratamiento. Se observó una relación significativa entre precocidad del tratamiento y respuesta favorable (Fisher $p<0,02$ ). Este esquema de tratamiento no produjo efectos 
laterales severos en paciente alguno. Durante el seguimiento ( $\overline{\mathrm{X}}: 6,2$ meses) se observó recaida en un caso de respuesta completa, en tanto que cinco pacientes mantienen su respuesta inicial con un desarrollo normal o levemente retarda. do. Los cinco pacientes restantes presentan un retardo severo y crisis de dificil tratamiento. Futuros estudios debieran abordar lá eficacia de otros esquemas de tratamiento, balanceando éxito y efectos laterales.

(Palabras clave: Espasmos infantiles, tratamiento, ACTH.)

\section{Agradecimientos}

Agradecemos al personal técnico del Departamento de Electroencefalografía del Servicio de Neuropsiquia. tría Infantil del Hospital San Borja-Arriarán pot su colaboración en este estudio.

\section{Referencias}

1. Sorel L, Dusaucy Bauloye A: A propos de 21 cas d'hypsarritmia de Gibbs. Son traitement spectacularie par l'ACTH. Acta Neurol Psychiatr Belg 1958; $58: 130 \cdot 141$.

2. Rikonen $\boldsymbol{R}$ : Infantile Spasms: modem practical aspects. Acta Paediatt Scand 1984; 73: 1-12.

3. Jeovons PM: West Syndrome: Infantile Spasms. En: Epileptic Syndromes in infancy, childhood and adolescence, eds: Dravet, Bureau, Dreifuss, Wolf. John Libbey, Eurotext, 1985; 42-50.

4. Aicardi 5 : Treatment of infantile spasms. En: Intractable Epilepsy. Eds. D Schmidt and PL Morselli. Raven Press. N.Y., 1985; 147-156.

5. Snead $O C$ : Treatment of infantile spasms. Pediatr Neurol 1990; 6: 147-150.

6. Hrachovy RA, frost JD. Kellawey $P$, Zion TE: Double blind study of ACTH vs prednisone therapy in infantile spasm. J Pediats 1983; 103: 641-645.

7. Riikonen $R$, Donner M: ACTH therapy in infantile spasms: side effects. Arch Dis Child 1980; 55: 664-672.
8. Devilat $M$, Valdivieso $A$, Latorre $M$ et al.: Encefalopatía mioclónica infantil con hipsarritmia. Rev Med Chile 1973; 101: 376-381.

9. Soret $L$ : 196 cases of infantile mioclonic encephalopaty with hypsirritmin treated with ACTH: danger of synthetic ACTH (Abstract). Electroenceph Cin Neurophsyiol 1972; 32: 576

10. Lerman $P$, Kivity $S$ : The efficacy of corticotropin in primary infantile spasms: J. Pediatr 1982; 101: 294-296.

11. Mosatoshi I, Takeniko $O$. Tasuya $F$ et al.: ACTH therapy in infantile spasms: Relationship between dose of ACTH and initial effect or long term prognosis. Ped Neurol 1990; 6: 240.244.

12. Synacthen Retard. Etat actuel de la therapeutique par I'ACTH. Ohimie et Pharmacologie du Synacthen et du Synachten Retard. Nov 1969. Archivos del Laboratorio CIBA-GEIGY.

13. Gibbs $F A$, Gibbs $E L$ : Atlas of electroencephalography. Vol. II. Epilepsy. Addison Wesley, Cambridge, Mass 1952; 24-25.

14. Hrachovy RA, Frost JD, Kellaway P: Hypsarrhythmia. Variation on the theme. Epilepsia 1984; 25: $317-325$.

15. Trvine WJ, Wilson $K S$, Taff $A D$ : Repercusiones hormonales de una terapéutica prolongada con preparado de ACTH sintético en: ACTH reseña práctica de conocimientos actuales. Ed. por $R$. Schuppli, Hans Huber, Berna. 1974; 46-60.

16. Stegel $S$ : Estadística no paramétrica. Editorial Tiillas, México. 1970; 120-130.

17. Hrachovy $R A$ and Frost JD: Infantile Spasms. Pediatr Clin North Am 1989; 36: 311 1-329.

18. Snead OC, Bentone JW, Hosey $L C$ et al.: Treatment of infantile spasms with high dose ACTH. Neurology 1989; 39: 1027-1031.

19. Hrachovy $R A$, Frost JD, Kellaway $P$ and Zion $P$ : Controlled study of ACTH thexapy in infantile spasms. Epilepsia 1980; 21: 631-636.

20. Singer WD, Rabe EF, Haller JS: The effect of ACTH therapy upon infantile spasms. J Pediatr $1980 ; 96: 485-489$

21. Glaze DG, Hrachovy RA, Frost $J D$, Zion $T E$, Bryon N: Computed tomography in infantile spasms: Effects of hormonal therapy. Pediats Neurol 1986;2:23-27. 